\title{
A Highly Sensitive Method for the Simultaneous UHPLC-MS/MS Analysis of Clonidine, Morphine, Midazolam and their Metabolites in Blood Plasma Using HFIP as the Eluent Additive
}

Rūta Veigure ${ }^{\mathrm{a}}$, Rudolf Aro ${ }^{\mathrm{a}}$, Tuuli Metsvaht ${ }^{\mathrm{b}}$, Joseph F Standing ${ }^{\mathrm{c}, \mathrm{d}}$, Irja Lutsar ${ }^{\mathrm{e}}$, Koit Herodes $^{\mathrm{a}}$, Karin Kipper ${ }^{\mathrm{a}, \mathrm{d} *}$ on behalf of the CloSed Consortium

${ }^{\text {a }}$ University of Tartu, Institute of Chemistry, 14a Ravila Street, 50411 Tartu, Estonia

${ }^{\mathrm{b}}$ Tartu University Hospital, Lunini 6, 51014 Tartu Estonia

${ }^{\mathrm{c}}$ Inflammation, Infection and Rheumatology Section, UCL Great Ormond Street Institute of

Child Health, 30 Guilford Street, London, WC1N 1EH, United Kingdom

${ }^{\mathrm{d}}$ Paediatric Infectious Diseases Research Group, Institute for Infection and Immunity, St. George's, University of London, Cranmer Terrace, London, SW17 0RE, United Kingdom

${ }^{\mathrm{e}}$ University of Tartu, Institute of Microbiology, 19 Ravila Street, 50411 Tartu, Estonia

* e-mail: karin.kipper@gmail.com; phone: +44 2087255881

\begin{abstract}
In intensive care units, the precise administration of sedatives and analgesics is crucial in order to avoid under- or over sedation and for appropriate pain control. Both can be harmful to the patient, causing side effects or pain and suffering. This is especially important in the case of pediatric patients, and dose-response relationships require studies using pharmacokineticpharmacodynamic modeling. The aim of this work was to develop and validate a rapid ultra-high performance liquid chromatographic-tandem mass spectrometric method for the analysis of three common sedative and analgesic agents: morphine, clonidine and midazolam, and their metabolites (morphine-3-glucuronide, morphine-6-glucuronide and 1'-hydroxymidazolam) in blood plasma at trace level concentrations. Low concentrations and low sampling volumes may be expected in pediatric patients; we report the lowest limit of quantification for all analytes as $0.05 \mathrm{ng} / \mathrm{mL}$ using
\end{abstract}


only $100 \mu \mathrm{L}$ of blood plasma. The analytes were separated chromatographically using the C18 column with the weak ion-pairing additive 1,1,1,3,3,3-hexafluoro-2-propanol and methanol. The method was fully validated and a matrix matched calibration range of $0.05-250 \mathrm{ng} / \mathrm{mL}$ was attained for all analytes In addition, between-day accuracy for all analytes remained within $93-108 \%$, and precision remained within $1.5-9.6 \%$ for all analytes at all concentration levels over the calibration range.

\section{Keywords}

UHPLC-MS/MS, chromatographic separation, morphine, clonidine, midazolam, hexafluoroisopropanol. 


\section{Introduction}

Sedation is commonly used in intensive care units (ICU), and there is an increasing recognition of the need to avoid over-sedation, study non-benzodiazepines (which may lead to withdrawal and tolerance) and ensure adequate analgesia according to individual needs [1]. Sedative and analgesic requirements of children admitted to neonatal or pediatric ICU are under-studied, meaning optimal dosing is unclear. Furthermore, the desire to avoid the potentially harmful effects of benzodiazepines by moving towards using alpha- 2 adrenergic receptor agonists such as clonidine [2] are hampered by a lack of data on efficacy, safety and pharmacokinetics in the pediatric population.

The CLON01 study (EudraCT 2014-003582-24) "Clonidine for Sedation of Pediatric Patients in the Intensive Care Unit" (CloSed study) is a multicenter double-blind, randomized, controlled trial funded by the European Commission Framework 7 program comparing clonidine with midazolam used for sedation in neonatal and pediatric ICU (the current standard of care). In addition to these sedative drugs patients in either arm of this study do also receive morphine as analgesic component. A secondary endpoint of the study is to collect and analyze pharmacokinetic samples for all three substances to support the development of dose guidelines for sedation in neonatal and pediatric ICU.

Clonidine stimulates alpha (2)-adrenoceptors in the central nervous system which results in lowering blood pressure and decreasing of heart rate [3]. Because of that clonidine is used as an antihypertensive drug, but it is also used for multiple other indications such as sedation and analgesia [4].

Midazolam is a short-acting benzodiazepine with hypnotic, anticonvulsant, sedative, musclerelaxant and anxiety preventing properties [5]. Midazolam is hydroxylated to its primary active metabolite - 1'-hydroxymidazolam (MiOH) [6], meaning quantification of both parent and metabolite will be important to investigate sedative activity and potential developmental differences in metabolic activity with age.

Morphine is a highly addictive analgesic [7]. About $56 \%$ of the morphine is metabolized to morphine-3-glucuronide (M3G), and about $10 \%$ to morphine-6-glucuronide (M6G) [8]. Both glucuronides are very hydrophilic, but M6G crosses the blood-brain barrier more readily and due to its different plasma-concentration profile as well as long brain extracellular fluid half-life, has 
been found a more potent analgesic than $\mathrm{M} 3 \mathrm{G}$ or even morphine [9]. As with midazolam, quantification of these metabolites will also be important.

The simultaneous quantitation of sedatives and analgesics and their active metabolites will allow complex evaluation of the pharmacokinetic/pharmacodynamic relationships and defining optimal dosing for sedation at the same time limiting sample volumes and resource needs.

In total, 10 liquid chromatography-mass spectrometry (LC-MS) methods were reviewed. The lowest limits of quantification (LLOQ) ranging from $2-9 \mathrm{ng} / \mathrm{mL}$ for morphine and its two major metabolites [7,10,11], $0.01-100 \mathrm{ng} / \mathrm{mL}$ for clonidine - but clonidine was the only single compound analyzed in these assays [3,4,12,13], $0.025-5 \mathrm{ng} / \mathrm{mL}$ for midazolam $[10,11,14,15]$ and $0.1-2.5 \mathrm{ng} / \mathrm{mL}$ for $\mathrm{MiOH}[10,15]$. The exception was the LLOQ of $50 \mathrm{fg} / \mathrm{mL}$ for midazolam and $0.25 \mathrm{pg} / \mathrm{mL}$ for $\mathrm{MiOH}[16]$.

Basic conditions for reversed phase (RP) separation are useful in the case of pharmaceutical analyte analyses since over $70 \%$ of them have basic properties, but only approximately $20 \%$ are acids [17]. Basic analytes are protonated if the eluent's $\mathrm{pH}$ is lower than the analyte's $\mathrm{p} K_{\mathrm{a}}$ value and thus have poor retention in RP conditions [17]. Fluoroalcohols like 1,1,1,3,3,3-hexafluoro-2propanol (HFIP, $\mathrm{p} K_{\mathrm{a}}=9.3$ [18]) can be used in liquid chromatography - electrospray ionization source - mass spectrometry (LC-ESI-MS) as a buffer additive for basic solutions. Moreover, HFIP alters the selectivity of chromatographic separation. Fluoroalcohols are strongly retained on the hydrophobic RP stationary phase and thus create a hydrophilic layer with hydrogen bond donor properties. Furthermore, in the mobile phase the anions of fluoroalcohols form ion pairs with protonated bases and thus enhance their retention on the already altered stationary phase. Acidic compounds, however, have to compete with fluoroalcohols on the stationary phase surface, which decreases their retention [19].

We aimed to develop a method suitable for quantifying low levels of sedatives and their metabolites in the limited sample volume conditions - from neonatal and pediatric patients' blood plasma samples. Sufficiently low LLOQ levels will be necessary for obtaining adequate pharmacokinetic data for the evaluation of optimal dosing in the future. 


\section{Materials and methods}

\subsection{Chemicals}

Standard substances and their respective stable isotope labeled internal standards (IS): M3G, M6G, morphine, clonidine, MiOH, midazolam, M3G-D3, M6G-D3, morphine-D6 and MiOH-D4 were obtained from Cerilliant (Texas, USA). Clonidine-D4 and midazolam-D 6 were obtained from the Toronto Research Chemicals Inc. (Toronto, Canada). Other reagents used: LC-MS Ultra chromasolv grade methanol (MeOH) from Sigma Aldrich (Missouri, USA), LC-MS grade formic acid from Sigma Aldrich (Missouri, USA), LC-MS grade ammonium hydroxide solution from Sigma Aldrich (Missouri, USA), LC-MS grade 1,1,1,3,3,3-hexafluoro-2-propanol (HFIP) from Sigma Aldrich (Missouri, USA). Water was purified $\left(18.2 \mathrm{M} \Omega \cdot \mathrm{cm}\right.$ at $25^{\circ} \mathrm{C}$ and and a total organic carbon (TOC) value $2-3 \mathrm{ppb}$ ) in-house using a Millipore Advantage A10 system from Millipore (Bedford, USA). Plasma and whole blood were purchased from Blood Bank of Tartu University Hospital.

\subsection{Sample preparation}

Protein precipitation was accomplished by adding $50 \mu \mathrm{L}$ methanol containing $10 \mathrm{ng} / \mathrm{mL}$ IS and $700 \mu \mathrm{L}$ of neat methanol to $100 \mu \mathrm{L}$ of each calibrator, quality control (QC) or sample. The resulting solution was mixed for $4 \mathrm{~min}$ in the Eppendorf MixMate mixer (Hamburg, Germany) and centrifuged at $30,000 \times \mathrm{g}$ for $10 \mathrm{~min}$ at $4{ }^{\circ} \mathrm{C}$ in the Eppendorf Centrifuge $5430 \mathrm{R}$ (Hamburg, Germany). The supernatant (approximately $850 \mu \mathrm{L}$ ) was transferred into a $2 \mathrm{~mL}$ Eppendorf polypropylene vial and evaporated to dryness using the Jouan RC 10-09 centrifugal evaporator (Saint-Herblain, France) at $8-10$ mbar pressure at $1200 \mathrm{rpm}$. Samples were reconstituted in 80 $\mu \mathrm{L}$ of water and methanol mixture $(8: 2, \mathrm{v} / \mathrm{v})$. An aliquot of $6 \mu \mathrm{L}$ was injected into the UHPLCMS/MS system.

\subsection{Chromatographic conditions}

The Agilent 1290 Infinity (Santa Clara, USA) UHPLC system consisted of a binary pump, a thermostated column compartment and an autosampler (set at $4{ }^{\circ} \mathrm{C}$ ). Analytes were separated using the Waters Acquity UPLC BEH C18 $(2.1 \times 100 \mathrm{~mm}, 1.7 \mu \mathrm{m})$ analytical column with the Waters VanGuard BEH C18 $(2.1 \times 5 \mathrm{~mm}, 1.7 \mu \mathrm{m})$ pre-column (Milford, USA), which were maintained at $30{ }^{\circ} \mathrm{C}$. To protect both column and pre-column from unnecessary blockages, an in-line filter was installed ahead of them. The mobile phase consisted of water (solvent A) containing $5 \mathrm{mM}$ HFIP (v/v) (at $\mathrm{pH} 9$, adjusted with ammonium hydroxide solution) and methanol (solvent B). 
The flow rate for the gradient elution was $250 \mu \mathrm{L} / \mathrm{min}$. The gradient started from $5 \%$ solvent B for the first minute, then was increased to $75 \%$ until the $3.7 \mathrm{~min}$ and kept at $75 \%$ until the 5.8 minute. Between the 5.8 and $5.9 \mathrm{~min}$, the $\mathrm{MeOH}$ content was increased to $100 \%$ and kept there until the $6.9 \mathrm{~min}$, after which it was decreased back to $5 \%$ in $0.1 \mathrm{~min}$ and kept at $5 \%$ for 3 min to allow the column to equilibrate.

\subsection{Mass spectrometry}

Detection of the analytes and internal standards was achieved with the Agilent 6495 Triple Quad mass spectrometer (Santa Clara, USA), equipped with an Agilent JetStream electrospray ionization source. The instrument was operated in the positive ionization multiple reaction monitoring (MRM) mode. For controlling the LC-MS system, the Agilent MassHunter Workstation software version B.07.00 was used. The Agilent MassHunter Quantitative Analysis software version B.07.00 was used to quantify the analytes.

The following mass analyzer settings were used: drying gas temperature $135{ }^{\circ} \mathrm{C}$, drying gas flow rate $13 \mathrm{~L} / \mathrm{min}$, nebulizer pressure $25 \mathrm{psi}(172 \mathrm{MPa})$, sheath gas temperature $400{ }^{\circ} \mathrm{C}$ and sheath gas flow $(11 \mathrm{~L} / \mathrm{min})$, capillary voltage $(2,500 \mathrm{~V})$ and nozzle voltage $(500 \mathrm{~V})$. iFunnel voltage in the high pressure region was $210 \mathrm{~V}$ and at low pressure it was $220 \mathrm{~V}$. Optimized collision energies for each analyte and the internal standard transitions are listed in Table 1. Fragmentation patterns for these analytes have been documented previously $[13,16,20]$ and support the chosen $m / z$ values for the method.

\section{$* * *$ Table 1 goes here ${ }^{* * *}$}

\subsection{Validation}

The method was fully validated according to the European Medicines Agency (EMA) guideline [21]. The linear range, the LLOQ, the method's within-day and between-day accuracy and precision were evaluated. Moreover, an estimation of the matrix effect, carry-over, selectivity and analyte stability under different storage conditions was conducted.

In order to evaluate selectivity, independent blank plasma samples were analyzed. The analysis of the double blank plasma sample (blank plasma without the addition of an internal standard during sample preparation) was conducted every time when calibration samples were analyzed in order to re-assure selectivity. 
The carry-over was evaluated by comparing the peak areas in the blank sample injected after the higher concentration sample with the peak areas of the LLOQ sample.

Accuracy and precision were evaluated with QC samples at four concentration levels by analyzing 5 independently prepared samples at each level in every analytical run. QC samples were spiked using separate standard solutions with appropriate dilutions which were quantified using the calibration samples. According to the EMA guideline [21] for accuracy, the measured concentration should be within $15 \%$ of the nominal value (20\% at the LLOQ concentration level). The criterion for the precision is $15 \%$ (20\% at the LLOQ level).

Matrix effects (MEs) were estimated according to the EMA guideline [21] and as described by Matuszewski et al. [22]. The ME was evaluated as the ratio of the signal from the post-extraction spiked sample to the standard solution (at the same concentration level). For the ME evaluation, the analytes in neat solvent (standard solution) and the post-extraction spiked samples were analyzed. MEs in MS detection occur due to the components of the sample matrix which have not been removed during sample preparation. In the case of blood plasma samples, the ME is caused mostly by phospholipids that either enhance (ME over $100 \%$ ) or suppress (ME under $100 \%)$ the analytical signal. In addition, the interference in assay performance while using hemolyzed and hyperlipidemic plasma was evaluated.

Freeze and thaw stability was assessed in order to take into account the possible degradation in the case of the accidental thawing of samples during transportation, but also the possible bias caused by degradation during the incurred sample reanalysis. In order to evaluate this, spiked plasma samples at three concentrations - LLOQ, three times lowest limit of quantification (3xLLOQ) and medium concentration (MED) were frozen in a freezer at $-80{ }^{\circ} \mathrm{C}$ and thawed at room temperature in three cycles. In the course of each cycle, the samples were kept at $-80{ }^{\circ} \mathrm{C}$ for at least $24 \mathrm{~h}$.

For the evaluation of bench-top stability, spiked plasma samples at four concentrations - LLOQ, 3xLLOQ, MED and upper limit of quantification (ULOQ), were kept at monitored room temperature $\left(22^{\circ} \mathrm{C} \pm 1^{\circ} \mathrm{C}\right)$ for $24 \mathrm{~h}$.

For the evaluation of 24-hour stability, the samples were kept in an autosampler at an average temperature of $4^{\circ} \mathrm{C}$. 
In addition, long term (9 months) stability tests were conducted, keeping low (LLOQ $0.05 \mathrm{ng} / \mathrm{mL}$ ) and high (ULOQ $200 \mathrm{ng} / \mathrm{mL}$ ) concentration samples in a freezer at $-20^{\circ} \mathrm{C}$ and $-80{ }^{\circ} \mathrm{C}$ in order to evaluate the most suitable storage conditions.

\section{Results and discussion}

For the method development and validation, no clinical study samples were analyzed.

\subsection{Method development and the chromatographic retention mechanism of the analytes}

\section{$* * *$ Figure 1 goes here $* * *$}

The analytes analyzed have basic properties - morphine's $\mathrm{p} K_{\mathrm{a}}=8.2$ [23], clonidine's $\mathrm{p} K_{\mathrm{a}}=8.05$ [24], MiOH's $\mathrm{p} K_{\mathrm{a}}$ is estimated to be 4.99 [25], midazolam's $\mathrm{p} K_{\mathrm{a}}=6.15$ [26] and the eluent's water phase additive HFIP is a weak acid $\left(\mathrm{p} K_{\mathrm{a}}=9.3\right.$ [18]), thus the eluent's $\mathrm{pH}$ was adjusted to $\mathrm{pH}=9$ using ammonium hydroxide. Glucuronides' $\mathrm{p} K_{\mathrm{a}}$ values should be similar to those of morphine, however they also have a carboxylic acid group with the $\mathrm{p} K_{\mathrm{a}}$ value ranging 3-4 [27].

HFIP was added to enhance the compounds' signal in mass spectrometric detection [28] and to allow alternative chromatographic separation for morphine, M3G and M6G. The two metabolites share the same MS transitions (Table 1) and must therefore be chromatographically separated.

For simultaneous analysis of morphine and its glucuronides, they must be chromatographically separated. Namely, M3G and M6G partly undergo in-source collision induced dissociation (CID) losing the glucuronide moiety, which leads to $m / z$ identical to that of morphine $(\mathrm{m} / \mathrm{z} 286)$. Without chromatographic separation, glucuronides would contribute to the signal of morphine [20].HFIP acts as a weak ion-pairing additive in the basic mobile phase and therefore provides alternative selectivity in the C18 stationary phase [19]. Due to the carboxylic acid group in M3G and M6G, it is completely deprotonated at the used eluent $\mathrm{pH}$ 9.0. As a result, analytes are ionized and therefore elute early from the chromatographic system. Alternative selectivity compared to the commonly used C18 stationary phase is provided by HFIP, which interacts strongly with RP and forms a fluorous layer on its surface.

Morphine and clonidine have similar $\mathrm{p} K_{\mathrm{a}}$ values, thus their complete chromatographic separation was challenging. Both compounds' $\mathrm{p} K_{\mathrm{a}}$ values are lower than the $\mathrm{pH}$ of the eluent and being basic compounds they are deprotonated (neutral), explaining longer retention times than those of glucuronides. The fluorinated stationary phase provides alternate selectivity for the 
fluorinated analytes [29] midazolam and $\mathrm{MiOH}$. Besides having a similar structure to midazolam, $\mathrm{MiOH}$ is more polar and it elutes faster.

\subsection{Method validation}

\subsubsection{Selectivity}

Selectivity was evaluated by analyzing 6 independent blank plasma samples. None of the analyzed plasma samples contained peaks on method transitions and retention times (Table 1).

\subsubsection{Carry-over}

During the method development, carry-over was observed. Extended needle and seal wash programs and multiple blank injections with prolonged washing with $100 \% \mathrm{MeOH}$ did not decrease the carry-over sufficiently. Injecting $0.1 \%$ formic acid solution decreased carry-over significantly, but not sufficiently, thus it was decided to completely change the eluent A to $0.1 \%$ formic acid solution for the wash program after the injection of high concentration samples. The acidic eluent was chosen to improve the solubility of basic analytes. In acidic conditions, basic analytes become protonated, thus they become polar and less retained on the non-polar C18 stationary phase or on the needle and seal. In order to further clean the system, the needle wash with $\mathrm{MeOH}$ and $0.1 \%$ formic acid 1:1 (v/v) was conducted. The wash program with $0.1 \%$ formic acid as the eluent A proved to be more efficient compared to the basic eluent. Due to the low concentration of IS, no carry-over was observed for them. The conducted clean-up gradient with $0.1 \%$ formic acid and $\mathrm{MeOH}$ helped to remove the carry-over for all analytes.

\subsubsection{The lower limit of quantification}

The LLOQ's achieved are listed in Table 2. The target LLOQ levels were achieved with the signal to noise ratio (s/n) 5 or higher (as determined by the Agilent MassHunter Qualitative Analysis software version B.06.00) and the LLOQ level accuracy and precision were within $20 \%$ - as required by the EMA guideline [21]. The LLOQ was additionally assessed with regard to accuracy and precision.

\subsubsection{Calibration curve}


The matrix matched calibration consisted of 10 concentration levels in addition to the blank and double blank samples and was analyzed in duplicates. The range $0.05-250 \mathrm{ng} / \mathrm{mL}$ was chosen to fit the expected concentrations in the CloSed clinical trial samples based on the previous studies and the aim of the clinical trial to lower the concentration of sedatives. The curve was constructed using linear regression with $1 / \mathrm{x}^{2}$ (for M6G, clonidine, $\mathrm{MiOH}$ and midazolam) and 1/x (for M3G and morphine) weighting, the squared regression coefficient for all analytes was > 0.9930 (Table 2). All calibration curve points were within the set accuracy limits of $85-115 \%$ [21] of their back-calculated values.

\subsubsection{Accuracy}

Accuracy is influenced by both the analyte and the concentration level at low concentrations when the noise level is more influential and peak shapes are often not ideal, while the range of the accuracy is wider. This is especially true for within-day accuracies at the LLOQ level ranging from $97 \%$ to $111 \%$ for $\mathrm{M} 3 \mathrm{G}$, from $100 \%$ to $113 \%$ for clonidine and from $87 \%$ to $101 \%$ for midazolam. Morphine's within-day accuracies varied in the range of $96-109 \%$ for the LLOQ QC level. Accuracies for M6G and MiOH ranged from $96 \%$ to $102 \%$ and from $102 \%$ to $108 \%$,

respectively, at the LLOQ level. Within-day accuracies at all other concentration levels for all analytes ranged from $87 \%$ to $110 \%$, except for M6G ranging from $85 \%$ to $103 \%$. In general, at the ULOQ concentration level, the QCs were slightly overestimated, except for M6G. The biggest variations in the M6G accuracies could have been caused by the non-ideal peak shape in the chromatograms. Between-day accuracies are presented in Table 2.

\section{****Table 2 goes here***}

\subsubsection{Precision}

Precision is expressed as the coefficient of variation (CV) [21]. Similarly to accuracy, also precision (between-day precision is presented in Table 2) depended on the analyte and concentration level, while low concentrations are more influenced and show higher variation. In this regard, especially M6G stands out with the CV being 9.6\% at the LLOQ level, which is also the largest among all the analytes at all concentration levels. Precision is affected by high noise levels at low concentrations of glucuronides. The variability of the within-day precision was larger, 
however trends remained the same - lower concentrations were affected more. The imprecision was highest for M6G at LLOQ concentration level: between $7.8-10 \%$. The smallest within-day variation at low concentration was observed for midazolam, ranging between $1.1-2.1 \%$. However, precision values (both within-day and between-day) remained within the allowed range, according to the guideline [21] for all of the compounds - below $15 \%$ for the $3 \times$ LLOQ, MED and ULOQ levels and below $20 \%$ for the LLOQ level.

\subsubsection{Matrix effect}

A stronger ME was observed at low concentration levels. M6G was influenced the most by the signal enhancement (ME $125 \%$ at the LLOQ level). The ME for morphine also indicated a signal enhancement at the LLOQ level with the ME of $117 \%$. For other compounds, signal suppression was observed in the range of $83-98 \%$. For all compounds at the 3xLLOQ level, the ME ranged from $106-118 \%$ except M3G which was at $98 \%$. At the MED level, the ME ranged from $95-$ $109 \%$ for all compounds (see supplementary data). The use of matrix matched calibration and the IS enabled the MEs to be accounted for.

In addition, hemolyzed and hyperlipidemic plasmas were tested (see supplementary data) in order to evaluate matrix effects. Hemolyzed plasma samples were prepared by spiking pure plasma with whole blood in the $0-10 \%$ range of whole blood content in the plasma. All analytes' concentrations were within 85 - $104 \%$ when compared to pure plasma samples at a low (at the LLOQ level, $0.05 \mathrm{ng} / \mathrm{mL}$ ) concentration. Results were not influenced by hemolysis at higher concentrations, ranging from $87 \%$ to $103 \%$. However, the influence of hemolysis was increased at a high concentration (at the ULOQ level, $200 \mathrm{ng} / \mathrm{mL}$ ) for $\mathrm{MiOH}$ when the whole blood content in the plasma sample was $5 \%$ and $7 \%$ (difference from the non-hemolyzed plasma was 19-20\%). The higher hemolysis rate is influencing the most determination of $\mathrm{MiOH}$ at the high concentration level and should be encountered while analyzing hemolyzed plasma. Hyperlipidemia did not influence accuracies strongly, the change was 87 - 103\% for LLOQ samples and $90-105 \%$ for ULOQ samples.

\subsubsection{Stability $(S T)$}

Substock stability. Stability of substocks (in water) were tested comparing freshly made substocks (in the concentration range $0.9 \mathrm{ng} / \mathrm{mL}-5 \mu \mathrm{g} / \mathrm{mL}$ ), and substocks which were kept in a 
freezer at $-80^{\circ} \mathrm{C}$ for 8 months. Only minimal differences in concentrations were observed. Both glucuronides and morphine remained $100-103 \%$ from their original concentrations and experienced no degradation over 8 months at $-80{ }^{\circ} \mathrm{C}$ (with the standard deviation of $4-6 \%$ over all compared concentrations). Clonidine, midazolam and $\mathrm{MiOH}$ retained $94-99 \%$ of the original content.

Freeze and thaw stability. Analytes at low concentrations were affected by repeated freezing and thawing the most, but virtually no influence was evident when the analytes' concentration was higher than $50 \mathrm{ng} / \mathrm{mL}$ (Figure 2). The biggest degradation occurred at the LLOQ level for morphine (only $76 \%$ of the initial concentration remained after three thawing cycles), and for M6G with 88 $\%$ of its initial concentration.

\section{***Figure 2 goes here****}

Short term stability or bench-top stability. Variations for all analytes at all four concentration levels were within $85-105 \%$ (see supplementary data), except for morphine at the LLOQ and 3xLLOQ concentration levels. Morphine's concentration after $24 \mathrm{~h}$ was $69-84 \%$ at low concentrations levels, but the standard deviation for these results was high. Based on the short term stability results, it was decided to decrease the period for which the samples were kept at room temperature to a minimum (up to 60 minutes as absolute maximum for clinical trial samples).

$24 \boldsymbol{h}$ stability in the autosampler at $4{ }^{\circ} \mathrm{C}$. Concentration variations were within $85-105 \%$ for all compounds at all four concentration levels (Table 3).

\section{***Table 3 goes here ${ }^{* * *}$}

\section{Long term stability at $-20^{\circ} \mathrm{C}$ and $-80^{\circ} \mathrm{C}$.}

Samples at the LLOQ concentration level (Figure 3) were stable for 9 months $(85-110 \%)$ when kept in a freezer at $-80^{\circ} \mathrm{C}$, which is in the range of permitted difference $(15 \%)$. Storage at $20{ }^{\circ} \mathrm{C}$ showed a bigger decrease $(82-103 \%)$ in the compounds' concentrations and larger variation in results. Previously published studies show that analytes were stable both in long and short term even if kept at $-20{ }^{\circ} \mathrm{C}$ for different periods of time, [3,7,30]; however, the published lower concentrations were significantly higher compared to our assay. Only a minimal decrease in 
concentrations was observed - for M3G and M6G by $7 \%$ and for morphine by $5 \%$ after 6 months of storage [7]. Clonidine was stable for 4 months - a decrease by $11 \%$ in low concentrations and by $2.6 \%$ in high concentrations was observed [3]. Midazolam and $\mathrm{MiOH}$ were stable in spiked plasma samples for at least 10 months [30], however no data were shown to support this claim.

\section{***Figure 3 goes here***}

Samples at the ULOQ concentration level (Figure 4) were not strongly influenced by different temperatures: $99-106 \%$ when kept in a freezer at $-80{ }^{\circ} \mathrm{C}$ and $97-106 \%$ when samples were kept in a freezer at $-20^{\circ} \mathrm{C}$. Based on the results from the LLOQ samples, we recommend strongly for the future studies to store all the study samples at $-80^{\circ} \mathrm{C}$ over the longer periods (such as 9 months) of time.

\section{***Figure 4 goes here***}

\section{Conclusions}

A highly sensitive simultaneous UHPLC-MS/MS method was developed for the simultaneous quantification of morphine, morphine-3- $\beta$-glucuronide, morphine-6- $\beta$-glucuronide, clonidine, midazolam and 1'-hydroximidazolam in human plasma samples. The use of HFIP as an eluent additive improved the chromatographic separation of basic and zwitterionic compounds (especially between structurally similar morphine glucuronides) and increased the signal intensity of analytes, helping to achieve required LLOQ levels of $0.05 \mathrm{ng} / \mathrm{mL}$ using only $100 \mu \mathrm{L}$ of blood plasma. Matrix effects were assessed for all compounds and were compensated with the usage of matrix matched calibration and stable isotope labeled internal standards for every analyte. It is essential to assess compound stability with subsequent consideration during different steps of clinical research, such as sampling, sample storage and transportation, as well as interpretation of results in the evaluation of pharmacokinetics.

The assay will be used to analyze pediatric patients' samples in the EU FP7 project CloSed "Clonidine for Sedation of Pediatric Patients in the Intensive Care Unit". 


\section{Acknowledgments}

This project has received funding from the European Union's Seventh Framework Programme for research, technological development and demonstration under grant agreement $n^{\circ} 602453$. The research leading to these results has received funding from the People Programme (Marie Curie Actions) of the European Union's Seventh Framework Programme (FP7/2007-2013) under REA grant agreement $n^{\circ}$ 608765. This work was also supported by PUTJD 22 from the Estonian Research Council and by the institutional research grant of Ministry of Education and Research of Estonia IUT20-14 (TLOKT14014I). Joseph F. Standing has received funding from the United Kingdom Medical Research Council Fellowships (grants G1002305 and M008665), and been supported by the National Institute for Health Research Biomedical Research Centre at Great Ormond Street Hospital for Children NHS Foundation Trust and University College London.

\section{Conflict of Interest}

The authors have no other relevant affiliations or financial involvement with any organization or entity with a financial interest in or financial conflict with the subject matter or materials discussed in the manuscript, apart from those disclosed. No writing assistance was utilized in the production of this manuscript.

\section{References}

[1] N. Pathmanathan, J. McClure, Sedation and delirium in the intensive care unit, Anaesth. Intensive Care Med. 17 (2016) 17-23. doi:10.1016/j.mpaic.2015.10.002.

[2] S. Arenas-López, S. Riphagen, S.M. Tibby, A. Durward, S. Tomlin, G. Davies, I. a Murdoch, Use of oral clonidine for sedation in ventilated paediatric intensive care patients., Intensive Care Med. 30 (2004) 1625-9. doi:10.1007/s00134-004-2319-0.

[3] S.A. Parekh, A. Pudage, S.S. Joshi, V. V Vaidya, N.A. Gomes, Rapid and sensitive liquid chromatography-tandem mass spectrometry (LC-MS/MS) method for the determination of clonidine in human plasma., J. Chromatogr. B. Analyt. Technol. Biomed. Life Sci. 867 (2008) 172-8. doi:10.1016/j.jchromb.2008.03.030.

[4] C. Müller, M. Ramic, S. Harlfinger, C. Hünseler, M. Theisohn, B. Roth, Sensitive and 
convenient method for the quantification of clonidine in serum of pediatric patients using liquid chromatography/tandem mass spectrometry, J. Chromatogr. A. 1139 (2007) 221227. doi:10.1016/j.chroma.2006.11.020.

[5] S.P. Nordt, R.F. Clark, Midazolam: A review of therapeutic uses and toxicity, J. Emerg. Med. 15 (1997) 357-365. doi:10.1016/S0736-4679(97)00022-X.

[6] D.S. Streetman, J.S. Bertino, A.N. Nafziger, Phenotyping of drug-metabolizing enzymes in adults: a review of in-vivo cytochrome P450 phenotyping probes., Pharmacogenetics. 10 (2000) 187-216.

[7] D. Sartori, T. Lewis, A. Breaud, W. Clarke, The development of a high-performance liquid chromatography-tandem mass spectrometric method for simultaneous quantification of morphine, morphine-3- $\beta$-glucuronide, morphine-6- $\beta$-glucuronide, hydromorphone, and normorphine in serum., Clin. Biochem. 48 (2015) 1283-1290. doi:10.1016/j.clinbiochem.2015.05.023.

[8] C. Skarke, J. Lötsch, Morphine Metabolites: Clinical Implications, Semin. Anesth. Perioper. Med. Pain. 21 (2002) 258-264.

[9] S. De Gregori, M. De Gregori, G.N. Ranzani, M. Allegri, C. Minella, M. Regazzi, Morphine metabolism, transport and brain disposition, Metab. Brain Dis. 27 (2012) 1-5. doi:10.1007/s11011-011-9274-6.

[10] S. Ghassabian, S.M. Moosavi, Y.G. Valero, K. Shekar, J.F. Fraser, M.T. Smith, Highthroughput assay for simultaneous quantification of the plasma concentrations of morphine, fentanyl, midazolam and their major metabolites using automated SPE coupled to LC-MS/MS, J. Chromatogr. B. 903 (2012) 126-133. doi:10.1016/j.jchromb.2012.07.005.

[11] M. Fisichella, S. Odoardi, S. Strano-Rossi, High-throughput dispersive liquid/liquid microextraction (DLLME) method for the rapid determination of drugs of abuse, benzodiazepines and other psychotropic medications in blood samples by liquid chromatography-tandem mass spectrometry (LC-MS/MS) and app, Microchem. J. 123 (2015) 33-41. doi:10.1016/j.microc.2015.05.009.

[12] H. Danafar, M. Hamidi, LC-MS Method for Studying the Pharmacokinetics and Bioequivalence of Clonidine Hydrochloride in Healthy Male Volunteers., Avicenna J. Med. Biotechnol. 8 (2016) 91-8. http://www.ncbi.nlm.nih.gov/pubmed/27141268 
(accessed May 15, 2016).

[13] R. Nirogi, V. Kandikere, K. Mudigonda, P. Komarneni, Liquid chromatography tandem mass spectrometry method for the quantification of clonidine with LLOQ of $10 \mathrm{pg} / \mathrm{mL}$ in human plasma., Biomed. Chromatogr. 22 (2008) 992-1000. doi:10.1002/bmc.1018.

[14] N.S. Nosseir, G. Michels, P. Binder, M.H.J. Wiesen, C. Müller, Simultaneous detection of ketamine, lorazepam, midazolam and sufentanil in human serum with liquid chromatography-tandem mass spectrometry for monitoring of analgosedation in critically ill patients., J. Chromatogr. B. Analyt. Technol. Biomed. Life Sci. 973C (2014) 133-141. doi:10.1016/j.jchromb.2014.10.006.

[15] V. a P. Jabor, E.B. Coelho, N. a G. Dos Santos, P.S. Bonato, V.L. Lanchote, A highly sensitive LC-MS-MS assay for analysis of midazolam and its major metabolite in human plasma: Applications to drug metabolism, J. Chromatogr. B Anal. Technol. Biomed. Life Sci. 822 (2005) 27-32. doi:10.1016/j.jchromb.2005.05.011.

[16] J. Burhenne, B. Halama, M. Maurer, K.D. Riedel, N. Hohmann, G. Mikus, W.E. Haefeli, Quantification of femtomolar concentrations of the CYP3A substrate midazolam and its main metabolite 1'-hydroxymidazolam in human plasma using ultra performance liquid chromatography coupled to tandem mass spectrometry, Anal. Bioanal. Chem. 402 (2012) 2439-2450. doi:10.1007/s00216-011-5675-y.

[17] I.J. Wells, Pharmaceutical Preformulation: The Physicochemical Properties of Drug Substances, Ellis Horwoord, New York, 1988.

[18] A. Apffel, J.A. Chakel, S. Fischer, K. Lichtenwalter, W.S. Hancock, Analysis of Oligonucleotides by HPLC-Electrospray Ionization Mass Spectrometry., Anal. Chem. 69 (1997) 1320-5. doi:10.1021/ac960916h.

[19] K. Kipper, K. Herodes, I. Leito, Fluoroalcohols as novel buffer components for basic buffer solutions for liquid chromatography electrospray ionization mass spectrometry: retention mechanisms., J. Chromatogr. A. 1218 (2011) 8175-80. doi:10.1016/j.chroma.2011.09.025.

[20] W. Naidong, J.W. Lee, X. Jiang, M. Wehling, J.D. Hulse, P.P. Lin, Simultaneous assay of morphine, morphine-3-glucuronide and morphine-6-glucuronide in human plasma using normal-phase liquid chromatography-tandem mass spectrometry with a silica column and an aqueous organic mobile phase, J. Chromatogr. B Biomed. Sci. Appl. 735 (1999) 255- 
269. doi:10.1016/S0378-4347(99)00429-6.

[21] Committee for Medicinal Products for Human Use (CHMP), European Medicines Agency (EMA) guideline on bioanalytical method validation, (2011).

http://www.ema.europa.eu/docs/en_GB/document_library/Scientific_guideline/2011/08/ WC500109686.pdf (accessed May 1, 2016).

[22] B.K. Matuszewski, M.L. Constanzer, C.M. Chavez-Eng, Strategies for the assessment of matrix effect in quantitative bioanalytical methods based on HPLC - MS/MS, Anal. Chem. 75 (2003) 3019-3030.

[23] P.M. Dewick, Essentials of organic chemistry : for students of pharmacy, medicinal chemistry and biological chemistry, J. Wiley, 2006.

[24] K. Kontturi, L. Murtomaki, Electrochemical determination of partition coefficients of drugs, J. Pharm. Sci. 81 (1992) 970-975. doi:10.1002/jps.2600811003.

[25] W.J. Wishart DS, Knox C, Guo AC, Shrivastava S, Hassanali M, Stothard P, Chang Z, DrugBank: a comprehensive resource for in silico drug discovery and exploration. Alphahydroxymidazolam, Nucleic Acids Res. (2006). http://www.hmdb.ca/metabolites/HMDB61089 (accessed May 25, 2016).

[26] A. Walser, L.E. Benjamin, T. Flynn, C. Mason, R. Schwartz, R.I. Fryer, Quinazolines and 1,4-benzodiazepines. Synthesis and reactions of imidazo [1,5-a][1,4]benzodiazepines, J. Org. Chem. 43 (n.d.) 936-944.

[27] J. Netriova, E. Blahova, Z. Johanesova, E. Brandsteterova, J. Lehotay, K. Serdt, J. Mocak, HPLC determination of morphine, morphine-3-glucuronide and morphine-6-glucuronide in human serum of oncological patients after administration of morphine drugs, Pharmazie. 61 (2006) 528-534.

[28] K. Kipper, K. Herodes, I. Leito, L. Nei, Two fluoroalcohols as components of basic buffers for liquid chromatography electrospray ionization mass spectrometric determination of antibiotic residues, Analyst. 136 (2011) 4587-4594. doi:10.1039/c1an15123a.

[29] E.L. Regalado, A.A. Makarov, R. McClain, M. Przybyciel, C.J. Welch, Search for improved fluorinated stationary phases for separation of fluorine-containing pharmaceuticals from their desfluoro analogs, J. Chromatogr. A. 1380 (2015) 45-54. doi:10.1016/j.chroma.2014.12.025. 
[30] M. Shimizu, T. Uno, H. Tamura, H. Kanazawa, I. Murakami, K. Sugawara, T. Tateishi, A developed determination of midazolam and 1'-hydroxymidazolam in plasma by liquid chromatography-mass spectrometry: Application of human pharmacokinetic study for measurement of CYP3A activity, J. Chromatogr. B. 847 (2007) 275-281. doi:10.1016/j.jchromb.2006.10.018. 
Table 1. Retention times, monitored transitions and respective collision energies (CE) for analytes and IS-s.

\begin{tabular}{|c|c|c|c|c|c|c|c|}
\hline Analyte & $\begin{array}{l}\text { Retention } \\
\text { time, } \\
\text { min }\end{array}$ & $m / z^{*}$ & $\begin{array}{c}\text { Quantifier, } \\
m / z\end{array}$ & $\begin{array}{l}\text { Quantifie } \\
\text { r CE, V }\end{array}$ & $\begin{array}{c}\text { Qualifier, } \\
\mathrm{m} / \mathrm{z}\end{array}$ & $\begin{array}{c}\text { Qualifier } \\
\text { CE, V }\end{array}$ & $\begin{array}{l}\text { References } \\
\text { for } \\
\text { transitions }\end{array}$ \\
\hline M3G & 3.50 & 462.2 & $286.0\left[\mathrm{M}+\mathrm{H}-\mathrm{C}_{6} \mathrm{H}_{9} \mathrm{O}_{6}\right]^{+}$ & 26 & 200.8 & 46 & {$[22]$} \\
\hline M3G-D 3 & 3.50 & 465.2 & 289.0 & 32 & - & & \\
\hline M6G & 3.95 & 462.2 & $286.0\left[\mathrm{M}+\mathrm{H}-\mathrm{C}_{6} \mathrm{H}_{9} \mathrm{O}_{6}\right]^{+}$ & 32 & 200.8 & 52 & {$[22]$} \\
\hline M6G-D 3 & 3.95 & 465.2 & 289.1 & 36 & - & & \\
\hline morphine & 4.80 & 286.2 & 152.0 & 64 & $165.1\left[\mathrm{C}_{8} \mathrm{H}_{11} \mathrm{~N}\right]^{+}$ & 49 & {$[22]$} \\
\hline morphine-D 6 & 4.80 & 292.2 & 152.0 & 64 & - & & \\
\hline clonidine & 4.91 & 230.0 & $44.0\left[\mathrm{C}_{2} \mathrm{H}_{6} \mathrm{~N}\right]^{+}$ & 29 & 212.9 & 28 & {$[13]$} \\
\hline clonidine- $\mathrm{D}_{4}$ & 4.91 & 234.1 & 48.2 & 32 & - & & \\
\hline $\mathrm{MiOH}$ & 5.70 & 342.1 & $203.0\left[\mathrm{C}_{10} \mathrm{H}_{6} \mathrm{ClN}_{3}\right]^{+}$ & 29 & 324.0 & 21 & {$[18]$} \\
\hline $\mathrm{MiOH}-\mathrm{D}_{4}$ & 5.70 & 346.1 & 202.9 & 29 & - & & \\
\hline midazolam & 6.06 & 326.1 & $291.1[\mathrm{M}+\mathrm{H}-\mathrm{Cl}]^{+}$ & 29 & 222.0 & 57 & {$[18]$} \\
\hline midazolam-D6 & 6.06 & 332.1 & 297.0 & 32 & - & & \\
\hline
\end{tabular}

$*$ All precursor ions were $[\mathrm{M}+\mathrm{H}]$ 
Table 2. Validation parameters of the quantitative performance of the used sample preparation and detection method for all analytes.

\begin{tabular}{|c|c|c|c|c|c|c|c|c|c|c|c|c|c|c|}
\hline \multirow[b]{2}{*}{ Analyte } & \multirow[b]{2}{*}{$\begin{array}{c}\text { Linearity } \\
\mathrm{R}^{2}\end{array}$} & \multirow[b]{2}{*}{ Weighting } & \multicolumn{3}{|c|}{ LLOQ } & \multicolumn{3}{|c|}{ 3xLLOQ } & \multicolumn{3}{|c|}{ MED } & \multicolumn{3}{|c|}{ ULOQ } \\
\hline & & & $\begin{array}{c}\text { Mean } \\
\text { calc. } \\
\text { conc } \\
\mathrm{ng} / \mathrm{mL}\end{array}$ & $\begin{array}{c}\text { Accuracy } \\
\%\end{array}$ & $\begin{array}{l}\text { Precision, } \\
\text { CV [\%] }\end{array}$ & $\begin{array}{c}\text { Mean } \\
\text { calc. } \\
\text { conc } \\
\mathrm{ng} / \mathrm{mL}\end{array}$ & $\begin{array}{c}\text { Accuracy } \\
\%\end{array}$ & $\begin{array}{l}\text { Precision, } \\
\text { CV [\%] }\end{array}$ & $\begin{array}{c}\text { Mean calc. } \\
\text { conc } \\
\mathrm{ng} / \mathrm{mL}\end{array}$ & $\begin{array}{c}\text { Accuracy } \\
\%\end{array}$ & $\begin{array}{l}\text { Precision } \\
\mathrm{CV}[\%]\end{array}$ & $\begin{array}{c}\text { Mean } \\
\text { calc. } \\
\text { conc } \\
\mathrm{ng} / \mathrm{mL}\end{array}$ & $\begin{array}{c}\text { Accuracy } \\
\%\end{array}$ & $\begin{array}{l}\text { Precision } \\
\mathrm{CV}[\%]\end{array}$ \\
\hline M3G & 0.9992 & $1 / x$ & 0.055 & 102 & 3.9 & 0.147 & 95 & 4.3 & 51 & 96 & 2.4 & 201 & 101 & 2.8 \\
\hline $\mathrm{M} 6 \mathrm{G}$ & 0.9935 & $1 / x^{2}$ & 0.053 & 99 & 9.6 & 0.155 & 101 & 5.2 & 48 & 91 & 5.5 & 183 & 87 & 2.5 \\
\hline morphine & 0.9989 & $1 / x$ & 0.055 & 102 & 4.3 & 0.155 & 101 & 3.6 & 52 & 99 & 2.7 & 205 & 102 & 3.2 \\
\hline clonidine & 0.9969 & $1 / x^{2}$ & 0.055 & 108 & 3.7 & 0.146 & 95 & 2.1 & 51 & 95 & 1.8 & 212 & 108 & 2.8 \\
\hline $\mathrm{MiOH}$ & 0.9939 & $1 / x^{2}$ & 0.055 & 104 & 3.4 & 0.156 & 102 & 2.1 & 56 & 106 & 2.2 & 222 & 110 & 2.7 \\
\hline midazolam & 0.9930 & $1 / \mathrm{x}^{2}$ & 0.049 & 93 & 2.6 & 0.136 & 89 & 1.5 & 47 & 89 & 1.6 & 212 & 110 & 2.8 \\
\hline
\end{tabular}


Table 3. Results for $24 \mathrm{~h}$ stability (ST\%) in the autosampler at $4{ }^{\circ} \mathrm{C}$ and at four concentration levels with standard deviation (SD).

\begin{tabular}{ccccccccc} 
& \multicolumn{2}{c}{ LLOQ } & \multicolumn{2}{c}{$3 x$ xLOQ } & \multicolumn{2}{c}{ MED } & \multicolumn{2}{c}{ ULOQ } \\
\cline { 2 - 9 } Analytes & ST\% & $\begin{array}{c}\text { SD, } \\
\%\end{array}$ & ST\% & $\begin{array}{c}\text { SD, } \\
\%\end{array}$ & ST\% & $\begin{array}{c}\text { SD, } \\
\%\end{array}$ & ST\% & $\begin{array}{c}\text { SD, } \\
\%\end{array}$ \\
\hline M3G & 92 & 5 & 101 & 4 & 102 & 3 & 102 & 2 \\
M6G & 91 & 15 & 97 & 8 & 100 & 4 & 103 & 2 \\
morphine & 85 & 11 & 86 & 4 & 89 & 4 & 89.2 & 1.4 \\
clonidine & 90 & 3 & 90 & 2 & 96.4 & 1.3 & 96.7 & 1.3 \\
MiOH & 103 & 3 & 98 & 2 & 98.7 & 1.2 & 104 & 4 \\
midazolam & 98 & 3 & 97 & 2 & 102 & 2 & 105 & 3
\end{tabular}


Fig. 1 A typical chromatogram obtained at analytes' concentration of $0.5 \mathrm{ng} / \mathrm{mL}$. (1 - M3G, 2 M6G, 3 - morphine, 4 - clonidine, 5 - MiOH, 6 - midazolam)

Fig. 2 Freeze and thaw stability for all analytes at three concentration levels (LLOQ - $50 \mathrm{pg} / \mathrm{mL}$, $3 x L L O Q-150 \mathrm{pg} / \mathrm{mL}$ and MED - $50 \mathrm{ng} / \mathrm{mL}$ ) with standard deviation

Fig. 3 Long term stability with standard deviation for all analytes at the LLOQ concentration level after 9 months

Fig. 4 Long term stability with standard deviation for all analytes at the ULOQ concentration level after 8 months 